\title{
COMPLETE URETHRAL DUPLICATION WITH EPISPADIAS IN A YOUNG MAN: A CASE REPORT
}

\section{SHAUKAT ALI KHAN}

Department of Urology, Rangpur Medical College, Rangpur, Bangladesh

\begin{abstract}
:
A 25 year male patient presented with the complaints of passage of urine through two openings on his penis, with deformities of penis since birth. After birth his parents noticed, when he micturates, urine comes through an opening on the proximal part of dorsal surface of penis in addition to the opening on the tip of glans penis. The penis is also a bit deformed and dorsal part of corona glandis is bifid. No treatment for the anomaly was taken. At puberty his testes, scrotum and other secondary sexual characteristics developed normally. He feels sexual attractions to opposite sex and experiences nocturnal emissions periodically. His penis erects on sexual stimulations, but bends upwards and at the peak of excitement orgasm also occurs and semen comes through the ventral orthotopic opening only. General physical examination was unremarkable, but external genitalial examination, shows, two external urethral meatus - one small pit like opening at the normal orthotopic position on the tip of glans penis and another on the dorsal surface of proximal penile shaft with epispadias and exposed urethral plate distal to this.. He had also dorsal chordee and ventral hooding with dorsal bifid glans penis. He had normally developed scrotum with normal both testes. Corpora cavernosa are developed but separated proximally. Spongiosa also developed and orthotopic anterior urethra can be palpated. Other systemic examinations revealed no abnormalities. Routine investigations like urine $R / E, C / S$, S.creatinine, blood sugar, $C B C$ were normal. X-ray $K U B$ revealed small separation of pubic symphysiis. USG of whole abdomen with KUB revealed significant PVR (77cc) with features of cystitis (sediments in bladder) with no other abnormality. RGU and MCU revealed duplicated urethra - with an accessory urethra arising from bladder neck, runs above the orthotopic one along the shaft terminating at the proximal part of dorsal penile shaft. The lower orthotopic urethra is normal with no stricture or abnormality except narrowing of the distal anterior urethra and external opening. Cystogram shows normal bladder with no VUR or divarticula except thickend wall. Urethrocystopic examination after dilatation of stenosed EUM of orthotopic (ventral) urethra showed normal anterior and posterior urethra containing sphincter and verumotanum and prostatic urethra opening into the bladder neck. Then cystoscope passed through the dorsal epispadiac urethra, which showed a less developed urethra, opening into the protruding bladder neck. Urinary bladder and ureteric orifices were normal. Then excision of accessory epispadiac urethra and the urethral plate done as high as possible near to bladder neck, followed by correction of chordee by ventral plication and repair of bifid corona glandis. He had an uneventful recovery, though having a residual dorsal chordee, which was corrected by a second operation after 18 months, when excision of residual stump of accessory dorsal urethra followed by dermal graft repair of dorsal corporal body done.
\end{abstract}

Key words: urethral duplication, Epispadias (dorsal), urethral duplication, sagittal urethral duplication.

Bangladesh J. Urol. 2017; 21(1): 48-51

Correspondance : Md Shaukat Ali Khan, Department of Urology, Rangpur Medical College, Rangpur. Bangladesh. emailshaukaturo@gmail.com 


\section{Introduction}

Urethral duplication is a rare congenital anomaly, affecting mainly boys, resulting from malformation of urogenital sinus. It can be epispadiac, hypospadiac, fusiform or Ytype, classified according to the position of ectopic urethra.[1]

The anomaly is detected due to the presence of symptoms such as two urinary streams, dysuria and other symptoms due to infection in an accessory urethra or voiding from perineum or any other abnormal sites .Symptomatic cases require treatment. A case of complete epispadiac duplication of urethra which manifested as epispadias with severe dorsal chordee and ventral hooding is presented here.

\section{Case report}

A 25 year male patient presented in a private hospital with the complaints of passage of urine through two openings on his penis, along with bending and deformities of penis since birth. After birth his parents noticed, when he micturates, urine comes through an opening on the proximal part of dorsal surface of penis in addition to the opening on the tip of glans. The penis is also a bit deformed and dorsal part of corona glandis is bifurcated. They consulted rural doctors but failed to receive any specialized treatment. At puberty his testes, scrotum and other secondary sexual characteristics developed normally. He feels sexual attractions to females and experiences nocturnal emissions periodically. His penis erects on sexual stimulations, but bends upwards and at the peak of excitement orgasm also occurs and semen comes through the ventral orthotopic urethral opening only. General physical examination was unremarkable, but examination of the external genitalia shows, two external urethral meatus - one small pit like opening at the normal orthotopic position on the tip of glans penis and another on the dorsal surface of proximal penile shaft with epispadias and distal to this exposed urethral plate on dorsal penile shaft. He had also dorsal chordee and ventral hooding with dorsal bifid glans penis. He had normally developed scrotum with both testes normal in size, shape and consistency. Corpora cavernosa are developed but separated proximally. Spongiosa also developed and orthotopic anterior urethra can be palpated. Other systemic examinations revealed no abnormalities. Routine investigations like urine R/E, C/S, S. creatinine, blood sugar, CBC were normal. X-ray KUB revealed small separation of pubic symphysiis. USG of whole abdomen with KUB revealed significant PVR $(77 \mathrm{cc})$ with features of cystitis (sediments in bladder) with no other abnormality. RGU and MCU revealed duplicated urethra -with an accessory urethra arising from the protruded bladder neck, runs above the orthotopic one, along the shaft terminating at the proximal part of dorsal penile shaft. The lower orthotopic urethra is normal with no stricture or abnormality except some narrowing of distal anterior urethra and the external opening. Cystogram shows normal bladder with no VUR or divarticula except thickend wall. Urethrocystopic examination after dilatation of stenosed EUM of orthotopic (ventral) urethra showed normal anterior and posterior urethra containing sphincter and verumotanum and prostatic urethra opening into the bladder neck. Then cystoscope passed through the dorsal epispadiac urethra , which showed a less developed urethra, opening into the protruding bladder neck. Urinary bladder and ureteric orifices were normal in appearance. Then excision of accessory epispadiac urethra and the urethral plate done as high as possible near to bladder neck, followed by correction of remaining dorsal chordee by ventral plication and repair of bifid corona glandis. He had an uneventful recovery, though having a residual dorsal chordee. The patient underwent a second operation for correction of chordee after 18 months, when excision of residual stump of accessory dorsal urethra followed by dermal graft repair of dorsal corporal body done for chordee.

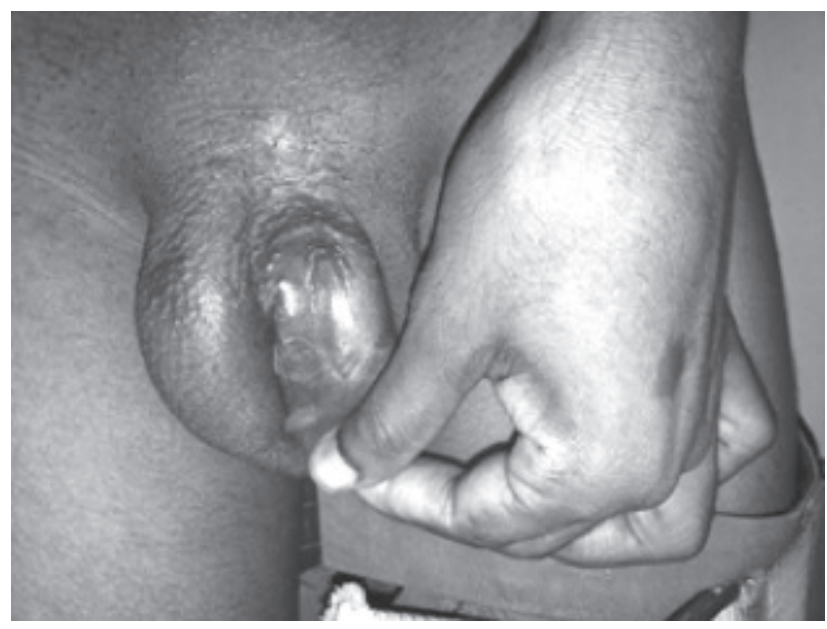

A. Dorsal accessory urethral opening with Epispadias at presentation 


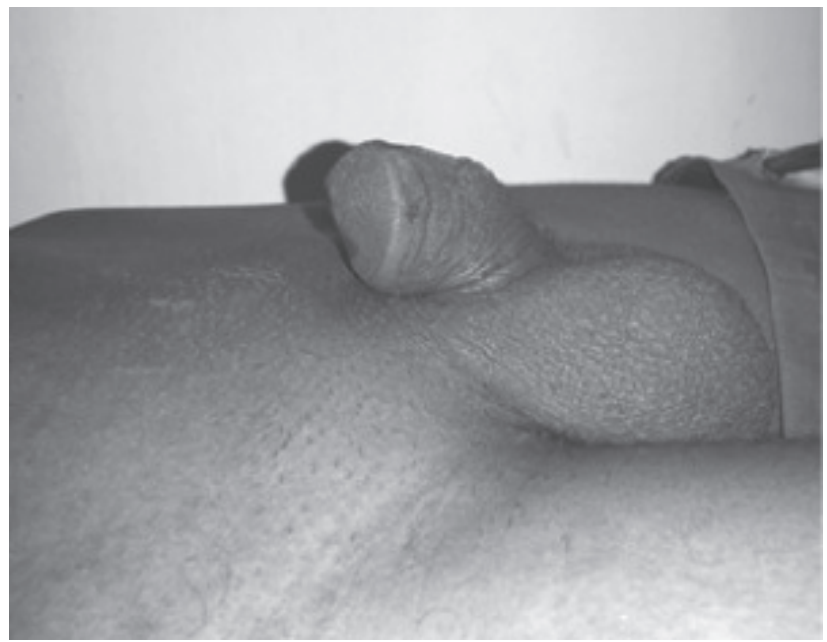

B. Dorsal Chordee at presentation

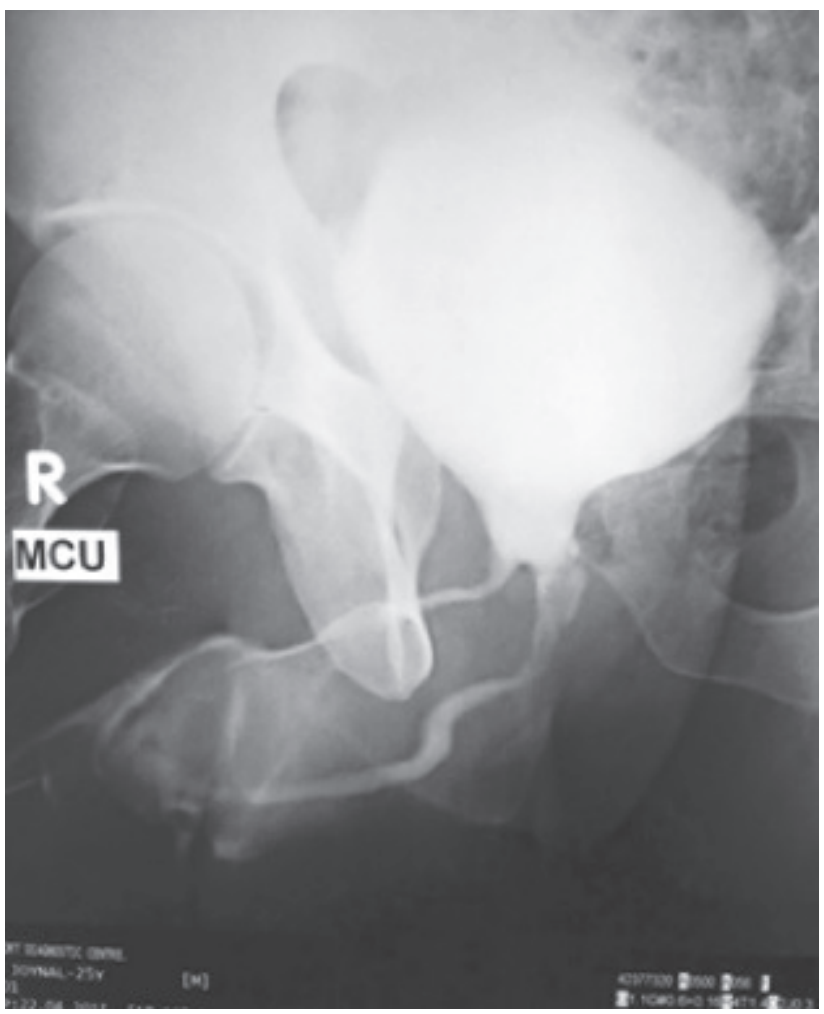

C. VCUG (MCU) at presentation showing complete urethral duplication (Type II-A-1) -2separate urethra arising from the protruding bladder neck.

\section{Discussion}

Urethral duplication is a rare anomaly with about 300 cases reported to date, usually seen in males and often associated with genitourinary and gastrointestinal anomalies.[2,3,4] Most urethral duplication are in the same sagittal plane, one on the top of the other [5] and less commonly in a frontal plane, lying with the normal

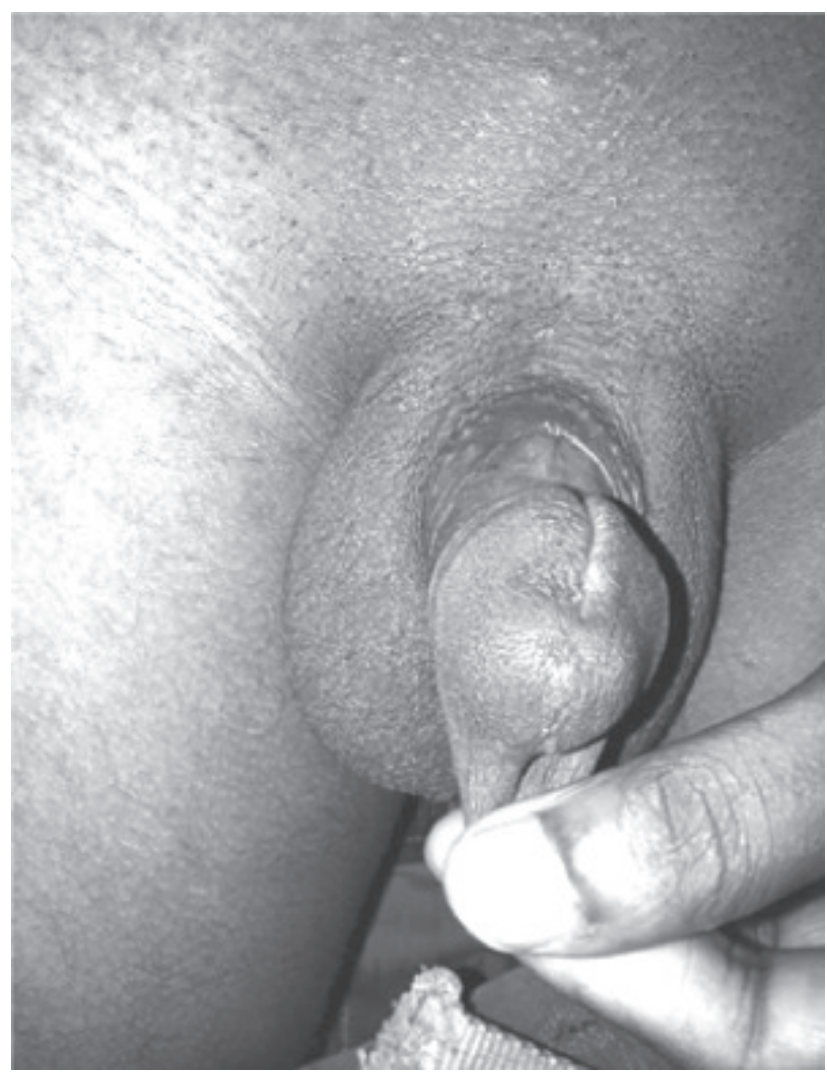

D. Ventral Hooding at presentation

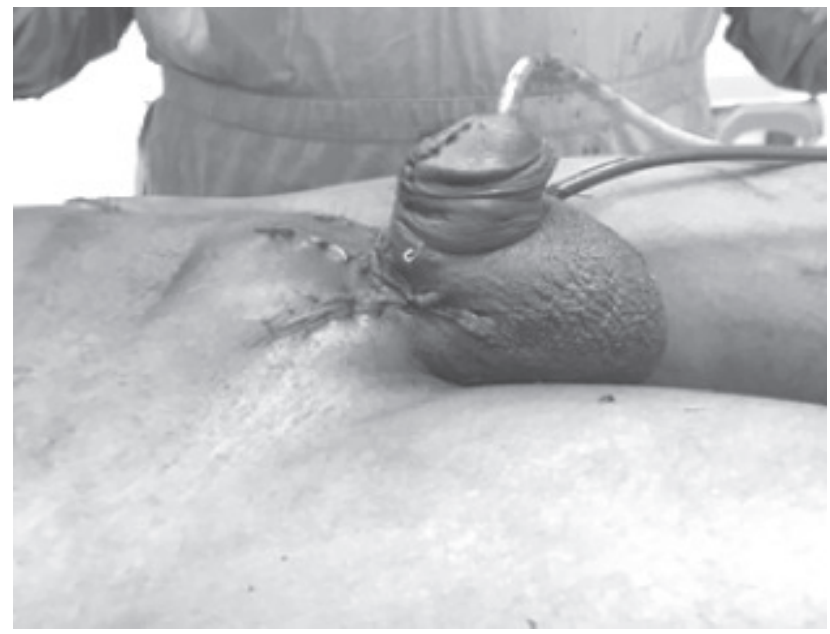

E. Immediate post operative appearance after $2^{\text {nd }}$ operation for Chordee

urethra. These may be complete or incomplete [6] .Complete duplicated urethra emerges from the bladder and opens somewhere on the penis or on the perineum. Incomplete variety may not originate from bladder and may not have an external opening. Complete urethral duplication is extremely rare, and can be ventral (hypospadiac) or dorsal (epispadiac). There are various 
types of classification, but the most complete classification has been offered by Effmann et al.[7] which shows three types-

Type I- blind incomplete duplication, where Type I Aduplicated urethra opens on penis but does not communicate with the urethra (most common), Type I B-duplicated urethra arise from original one and ends blindly in the periurethral tissue (rare).(8)

Type II- complete urethral duplication- Type IIA-1- two independent urethra arising from bladder opening with two meatus, Type IIA-2- single proximal channel opening in double streams. Type II B- two urethra opening in a single meatus.

Type III- urethral duplication is a component of caudal duplication .(8)

Features of urethral duplication may be two urinary streams, symptoms due to infection in accessory urethra. In epispadiac duplication dorsal chordee and irritative symptoms at meatus may be present. For preoperative evaluation, besides routine investigations determination of the length of the defect by RGU is done .VCUG may be diagnostic in complete urethral duplication.

The present case was one of the classical epispadiac urethral duplication with severe dorsal chordee with ventral hooding without incontinence. The orthotopic urethral opening was represented only by a indentation at the glanular tip. The epispadiac urethra had a separate origin from the bladder neck and it was going behind the pubic symphysis directly upwards. Thus it was urethral duplication Type IIA (1). The orthotopic urethra was normal except narrow external meatus and distal anterior urethra ,which needed dilatation before urethrocystoscopy. There was mild separation of symphysis pubis, which was observed by the earlier authors also,(7)

Treatment is indicated in symptomatic forms only. Blind ending duplications do not require excision. Incomplete forms require crushing and division of the common septum, for larger septa visual urethrotomy is needed .Epispadiac duplication along with dorsal chordee requires excision of epipadiac urethra with release of dorsal chordee after dissection upto penile base . In complete duplication treatment is joining the two tubes distally to allow a single stream. Pippe Salle et al (9) reported 5 males with a single bladder extrophy and complete urethral duplication, only one patient had the preoperative diagnosis of associated urethral duplication. In others, the duplicate ventral urethra was detected at the time of epispadias or fistula repair. The issue of deciding which urethra is the better of the two is very crucial. In sagittal duplications, if a preoperative diagnosis is available with
VCUG or retrograde urethrogram, the urethra with bigger caliber can be identified. In almost every instance ventral urethra is the normal variant. (10) If two streams can be seen, the urethra which brings out the better stream is chosen for preservation.

\section{Conclusion}

Urethral duplication is a rare congenital anomaly; either isolated or associated with other anomalies, with varied presentations and requires radiologic and endoscopic workup to define the anatomy and type to plan the surgical approach to provide cosmetically and functionally normal penis.

\section{Conflict of interested: None declared}

\section{References}

1. Batone FF,king LR Abnormalities of the urethra, penis and scrotum in Welch KJ, Randolph JG, Ravitch MM et al(eds), Pediatric surgery $4^{\text {th }}$ edn Volll Chicago,London, Year Book Medical publishers,INC,1986 pp 1322.

2. Arena S.,Arena C,Scuderi MG,Sanges G.Arena $\mathrm{F}, \mathrm{Di}$ BenedettoV.Urethral in males:Our experiences in ten cases.Pediatr surg Int.2007;23:789-4.[PubMed]

3. Mane SB Obaidah A,Dhende NP,Arlikar J,Acharya $\mathrm{H}$, Thakur $\mathrm{A}$,et al. Urethral duplication in children:Our experiences of eight cases.J.Pediatr Urol.2009;5:363-7.[PubMed]

4. Bhaduri S,Parashari UC,Shing R, Kohli N, Mri in congenital duplication of urethra. Indian J. Radiol Imaging.2009;19:232-4.[PMC free article] [PubMed]

5.. Haleblian G,Kraklau D, Wilcox D, DuffyP, Ransley $\mathrm{P}$, Mushtaq I,Y-type urethral duplication in male.BJU Int 2006;97:597-602.

6. Chen $\mathrm{CH}$,Chen JT,Hsieh JT,Epispadic urethral duplication in an adult dorsal deviated penis.Urology.2008;71(2):353.

7. Effman EL, Lebowitz RL, Colodny AH. Duplication of urethra. Radiology.1976;119: 179-183.[PubMed].

8. Al-Salem A, Incomplete urethral duplication simulating epispadias in anewborn.J Pediatr Surg Spec. 2008;2(3):50-51.

9. Pippi sale JL, Sibai H, Jacobson Al,et al. Bladder extrophy associated with complete urethral duplication-a rare malformation with excellent prognosis. J Urol 2001.165 2434-2437.

10. Monot $\mathrm{T}$, Weber $\mathrm{D}$,Stoyean $\mathrm{H}$,et al Urethral sagittal duplication in children-Report of 7 cases. Prog Urol 2002;12:77-83 